\title{
Erratum: Adiabatic excitation of longitudinal bunch shape oscillations [Phys. Rev. ST Accel. Beams 3, 064001 (2000)]
}

\author{
M. Bai, K. A. Brown, W. Fischer, T. Roser, N. Tsoupas, and J. van Zeijts \\ (Published 12 December 2000)
}

Corrections to our original manuscript are given in Table I, as well as other information which readers may find relevant. None of our conclusions are affected by these corrections.

In paragraph three, page 3 of our original article, the values of $\nu_{s}$ and $\nu_{m}$ need to be divided by 1000 to give the correct values.

TABLE I. Parameters in the AGS experiment.

\begin{tabular}{lccc}
\hline \hline \multicolumn{1}{c}{ Parameter } & Symbol & Unit & Value \\
\hline Species & $\ldots$ & $\ldots$ & $\mathrm{p}$ \\
Energy & $E$ & $\mathrm{GeV}$ & 24 \\
Harmonic number & $h$ & $\ldots$ & 6 \\
Number of bunches & $\ldots$ & $\ldots$ & 1 \\
Particles per bunch & $\ldots$ & $\ldots$ & $5 \times 10^{12}$ \\
rms bunch area & $\epsilon_{s}$ & $\mathrm{eV} \mathrm{s}$ & 4 \\
Slip factor & $\eta$ & $\ldots$ & 0.0122 \\
Peak gap voltage & $V_{\max }$ & $\mathrm{kV}$ & 190 \\
〈Gap voltage & $V_{0}$ & $\mathrm{kV}$ & 100 \\
Synchrotron tune & $\nu_{s}$ & $\ldots$ & 0.00022 \\
Modulation tunes & $\nu_{m}$ & $\ldots$ & $0.000404-0.000553$ \\
Gamma tr & $\gamma_{\text {tr }}$ & $\ldots$ & 8.5 \\
Longitudinal impedance & $Z_{\|} / n$ & $\Omega$ & 6 \\
Microwave cutoff (long) & $f_{c}$ long & $\mathrm{GHz}$ & $1.2-1.5$ \\
Microwave cutoff (trans.) & $f_{c}$ trans & $\mathrm{GHz}$ & $0.9-1.1$ \\
\hline \hline
\end{tabular}

